\title{
Electrostatic effects in coupled quantum dot-point contact-single electron transistor devices
}

\author{
S. Pelling, ${ }^{1}$ E. Otto ${ }^{2}$ S. Spasov, ${ }^{1}$ S. Kubatkin, ${ }^{2}$ R. Shaikhaidarov, ${ }^{1}$ K. Ueda,${ }^{3}$ S. Komiyama, ${ }^{3}$ \\ and V. N. Antonov ${ }^{1, a)}$ \\ ${ }^{1}$ Physics Department, Royal Holloway University of London, Egham, Surrey TW20 OEX, United Kingdom \\ ${ }^{2}$ Department of Microtechnology and Nanoscience (MC2), Chalmers University of Technology, \\ S-41296 Göteborg, Sweden \\ ${ }^{3}$ Department of Basic Science, University of Tokyo, Komaba 3-8-1, Meguro-ku, Tokyo 153-8902, Japan
}

(Received 6 February 2012; accepted 7 June 2012; published online 12 July 2012)

\begin{abstract}
We study the operation of a system where quantum dot (QD) and point contact (PC) defined in a two-dimensional electron gas of a high-mobility GaAs/AlGaAs heterostructure are capacitively coupled to each other and to metallic single electron transistor (SET). The charge state of the quantum dot can be probed by the point contact or single electron transistor. These can be used for sensitive detection of terahertz radiation. In this work, we explore an electrostatic model of the system. From the model, we determine the sensitivity of the point contact and the single electron transistor to the charge excitation of the quantum dot. Nearly periodic oscillations of the point contact conductance are observed in the vicinity of pinch-off voltage. They can be attributed to Coulomb blockade effect in a quasi-1D channel because of unintentional formation of small quantum dot. The latter can be a result of fluctuations in GaAs quantum well thickness. (C) 2012 American Institute of Physics. [http://dx.doi.org/10.1063/1.4736419]
\end{abstract}

\section{INTRODUCTION}

Recently, quantum dots (QD), point contacts (PC), and single electron transistors (SET) have been intensively exploited in applications to single-electron transport, quantization of conductance, and quantum computing. ${ }^{1-4}$ The devices have well defined quantum states with a typical energy scale of a few meV, which can be exploited for quantum information processing. ${ }^{5}$ Also all devices, QD, SET, and PC, have a high charge sensitivity, better than $10^{-4} e / \mathrm{Hz}^{1 / 2}$ at low temperatures. This opens the possibility to use them as electrometers, which are able to detect the motion of individual electrons. ${ }^{6,7}$ We studied coupled system of QD, PC, and metallic SETs in application to terahertz detection. The SET and the PC probe the charge state of the QD, which is varied by the absorption of the terahertz photons. A single photon counting with the SET-QD detector has been achieved earlier. ${ }^{8}$ The PC-QD detector is less sensitive, but it has the advantage of fewer technological demands in fabrication, and a higher operation temperature. ${ }^{9}$ We combine two devices together, SET-QD and PC-QD, in order to study electrostatic effects of the devices and compare their performance, see Fig. 1. An interesting phenomenon observed in PC-QD is nearly periodic oscillations of the source-drain conductance of the PC in the vicinity of pinch-off. The oscillations boost the sensitivity of the PC-QD terahertz detector because of the large trans-conductance $d G / d V_{g}$. At the same time, the effect makes the photo sensitive operation to be intermittent and strongly dependent on the operation point of $V_{g}$. We show that the oscillations occur because of the formation of

\footnotetext{
a) Author to whom correspondence should be addressed. Electronic mail: v.antonov@rhul.ac.uk.
}

a small dot (SD) in the PC channel, which operates in the regime of Coulomb blockade (CB) of electron tunnelling.

The samples consist of a mesa patterned GaAs/AlGaAs hetero-structure with two metal gates, PC gate and QD gate, and SET at the top of QD, see Fig. 1. The hetero-structure has been grown by molecular beam epitaxy. The layer sequence is $0.4 \mu \mathrm{m}$ super-lattice $\mathrm{GaAs} / \mathrm{Ga}_{0.3} \mathrm{Al}_{0.7} \mathrm{As}$ buffer, $20 \mathrm{~nm}$ GaAs well, $20 \mathrm{~nm} \mathrm{Ga}_{0.7} \mathrm{Al}_{0.3}$ As barrier layer, $60 \mathrm{~nm} \mathrm{Ga}_{0.7} \mathrm{Al}_{0.3} \mathrm{As}$ doping layer with a Si concentration of $1 \times 10^{18} \mathrm{~cm}^{-3}$, and $10 \mathrm{~nm}$ undoped GaAs cap layer. The two-dimensional electron gas (2DEG) is formed $90 \mathrm{~nm}$ below surface in GaAs well. It has carrier concentration $n=3.7 \times 10^{11} \mathrm{~cm}^{-2}$ and mobility $\mu=1.2 \times 10^{5} \mathrm{~cm}^{2} / \mathrm{Vs}$ at $\mathrm{T}=4.2 \mathrm{~K}$. The QD is formed in 2DEG by negatively biased QD gate. Aluminium SET is fabricated above the QD by two-angle deposition-oxidationdeposition technique. ${ }^{10}$

\section{OPERATION OF QUANTUM DOT-POINT CONTACT DEVICE}

One can probe charge state of the QD either with the PC or the SET. We start with the data taken by the PC probe. We negatively bias the gates and measure the conductance $G=I_{S D} / V_{S D}$ of mesa channel, see Fig. 2(a). The intensity plot is a compilation of individual curves $G\left(V_{Q D}\right)$ measured at constant voltage applied to the $\mathrm{PC}$ gate, $V_{P C}$, while sweeping the voltage, $V_{Q D}$, applied to the QD gate. The channel is pinched-off when a large negative bias is applied to both gates. There is asymmetry in the pinch-off boundary due to the difference in the PC and QD gate's geometry. Note also that the pinch-off boundary is not a straight line. The slopes of the pinch-off boundary are identical in regions $a$ and $c$, while it is larger in region $b$, indicating higher sensitivity of the PC conductance to the QD gate voltage, see Fig. 2(a). 


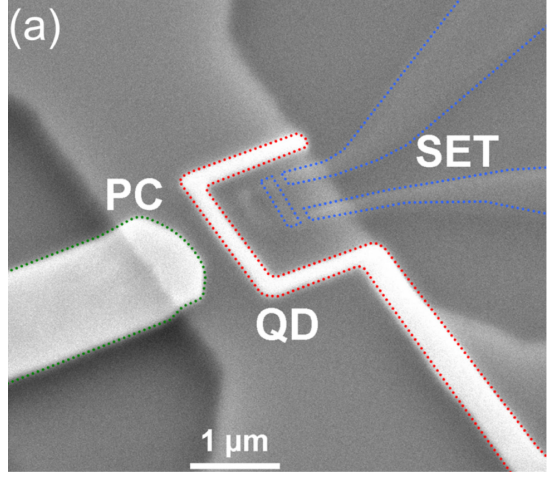

(b)

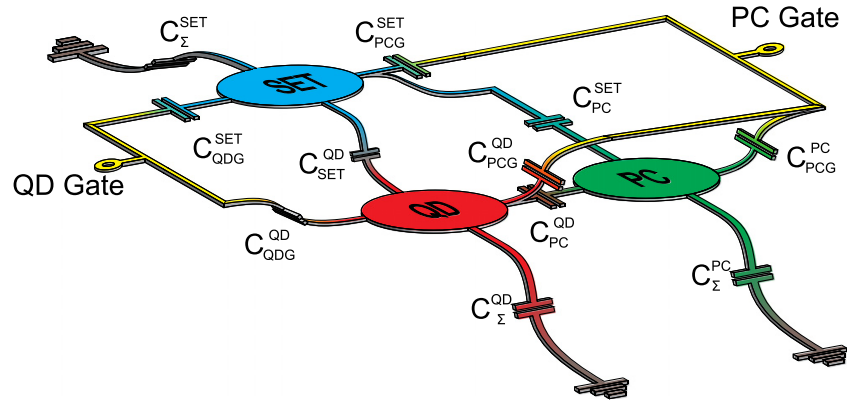

FIG. 1. (a) Scanning electron microscope image of the $\mathrm{THz}$ quantum dot sensor, consisting of the QD coupled to SET and PC. Dotted lines mark the QD gate, the SET, and the PC gate. The SET and PC senses charge excitations of the QD upon photon absorption, (b) electrostatic circuit model of the device.

The change of the slope can be expressed in terms of the change of the ratio, $C_{P C G}^{P C} / C_{Q D G}^{P C}$, from 2.1 in regions $a$ and $c$ to 0.78 in region $b$, where $C_{Q D G}^{P C}$ and $C_{P C G}^{P C}$ are capacitances of the $\mathrm{PC}$ to $\mathrm{QD}$ gate and to $\mathrm{PC}$ gate, respectively. In region $b$, the conductance channel is formed exactly between the $\mathrm{PC}$ and QD gates. This is the region where system can be used as terahertz detector. ${ }^{9}$ The QD is gradually isolated from reservoirs when the QD gate is negatively biased. This increases the effective capacitance $C_{Q D G}^{P C}$, because of additional parallel capacitance between the QD gate and the PC through the isolated QD. The white dashed line in Fig. 2(a) marks the boundary where the QD is isolated from the reservoirs. The line is taken from experiments with the SET, which is discussed below. In region $a$, the conductance channel is shifted in direction underneath the QD gate, so that the QD is formed simultaneously with a pinch-off of the conductivity. In region $c$, the conductance channel is pushed underneath the PC gate. The QD is already isolated from the reservoirs, because $V_{Q D}$ is beyond the dashed white line. An additional capacitance of the QD gate to the conductance channel, because of the isolated QD, is small since the QD and conductance channel are now spatially separated from each other. Therefore, one would expect that the slopes of the pinch-off boundary in regions $a$ and $c$ are almost identical.

Periodic oscillations of the PC conductance are observed close to the pinch-off boundary in region $b$, see Fig. 2(b). They were present in more than a half of 14 devices made of
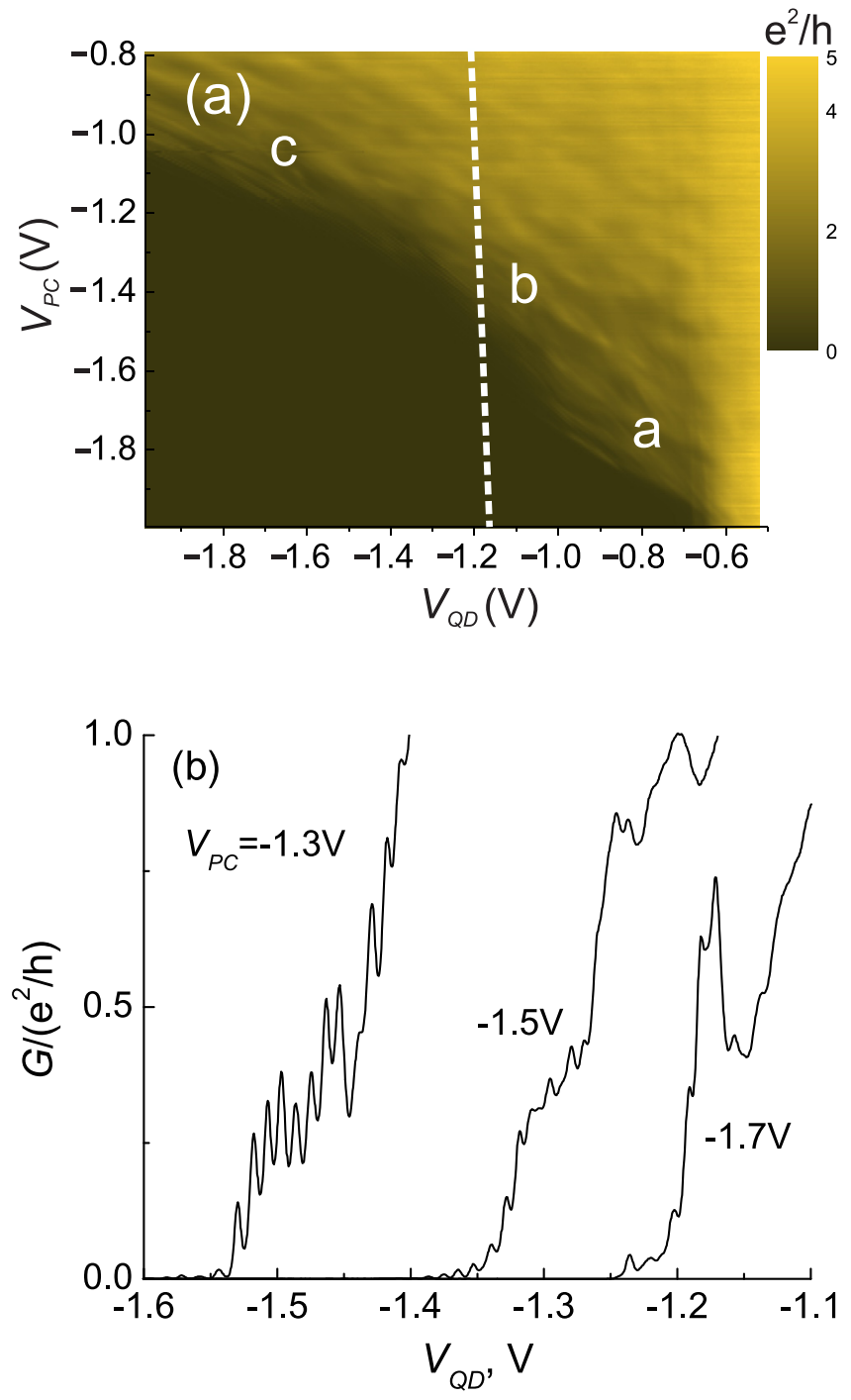

FIG. 2. (a) Intensity plot of $G$ in coordinates of $V_{P C}$ and $V_{Q D}$ close to pinchoff region. There are three distinct regions of PC operation marked by $a, b$, and $c$. In regions $a$ and $c$, the pinch-off boundary is a straight line with the identical slopes, in region $b$ the slope is larger. The white dashed line in the plot indicates the boundary separating isolated (left) and strongly coupled to reservoirs (right) QD. This boundary is taken from analysis of the SET operation. (b) Periodic oscillations of conductance $G$ are observed when crossing the pinch-off boundary. The PC gate voltage was fixed to $-1.3 \mathrm{~V},-1.5 \mathrm{~V}$, and $-1.7 \mathrm{~V}$. The oscillations are strongest in region $b$ and weaken in regions $a$ and $c$.

the same GaAs/AlGaAs wafer. The oscillations were highly reproducible for the particular sample. The other samples had a smooth pinch-off curve. In a sample presented here, the oscillation amplitude is largest at $V_{P C} \sim-1.3 \mathrm{~V}$ and $V_{Q D} \sim-1.47 \mathrm{~V}$. They weaken and disappear deep in regions $a$ and $c$. One can see from Fig. 2(b) that periodicity is not related to the conductance quantization of $\mathrm{PC}$ which was a subject of study in a number of works, ${ }^{11-13}$ as the position and number of oscillations are not correlated with the conductance quantum plateaus at multiples or rational numbers of $e^{2} / h$. There are more than ten periods of oscillations when $G$ is below $e^{2} / h$. In order to rule out possible quantum interference effects of the scattered electron waves, we have confirmed that oscillations are not sensitive to the magnetic field up to $1 \mathrm{~T}$. Observations of similar effect, periodic and 
aperiodic oscillations, in the conductance of narrow channels in 2DEG in GaAs/AlGaAs and $\mathrm{Si}$ quasi-1D systems have been reported before. ${ }^{14-16}$ The nearly periodic oscillations in conductance of Si quasi-1D channels were explained by the charge density wave, ${ }^{14}$ while a similar effect in an experiment with intentional channel doping by phosphorus atoms was attributed to the Coulomb blockade of electrons tunnelling between quantum dots formed around phosphorus atoms. ${ }^{15}$ In experiments with GaAs/AlGaAs heterostructures, the periodic oscillations were explained by the interference of scattered electron waves in quasi-1D channels. ${ }^{16}$

We present arguments in favour of the Coulomb blockade origin of the oscillation effect. Stability diagram of the second derivative of source-drain current $-d^{2} I_{S D} / d V_{S D}^{2}$ vs both $V_{S D}$ and $V_{P C}$ at fixed $V_{Q D} \sim-1.5 \mathrm{~V}$ is shown in Fig. 3. The map does not have a monotonous slope, so that the features of the pinch- off region are clearly seen. There is a diamond-shape structure marked by dashed white lines in Fig. 3. It can be explained either by the Coulomb blockade, or trans-conductance of the point contact in a regime of bound states. ${ }^{11,12}$ In a latter case, the diamonds reflect quantization of conductance of the PC: differential conductance has peaks when transition between different conductance plateaus occurs. In our samples, oscillations are not correlated with integer plateaus or, recently discussed, 0.5, 0.7, and 0.9 ones of $e^{2} / h$ in the $G$, see Fig. 2(b).

We believe that a SD of electrons is formed inside the $\mathrm{PC}$ conductance channel, which is in a regime of Coulomb blockade. From the horizontal diagonal of the diamonds, we estimate the charging energy of the dot, $E_{C} \sim 3 \mathrm{meV}$. This corresponds to diameter, $d=e^{2} /\left[4 \varepsilon_{0}\left(\varepsilon_{r}+1\right) E_{C}\right] \sim 100 \mathrm{~nm}$, if one models SD as a circular disk inside the conductance channel, $\varepsilon_{r}=13$ is the relative permittivity of GaAs, $\varepsilon_{0}$ is the permittivity of free space. Such a dot can be accommodated in a channel between the QD and the PC gates, which has a width of the same order $\sim 200 \mathrm{~nm}$. We get, however, a much smaller size of the dot from the analysis of conductance oscillations: in a wide range of $V_{Q D}$ the period of oscillations $\Delta V_{Q D} \sim 14 \mathrm{mV}$, which corresponds to a capacitance between

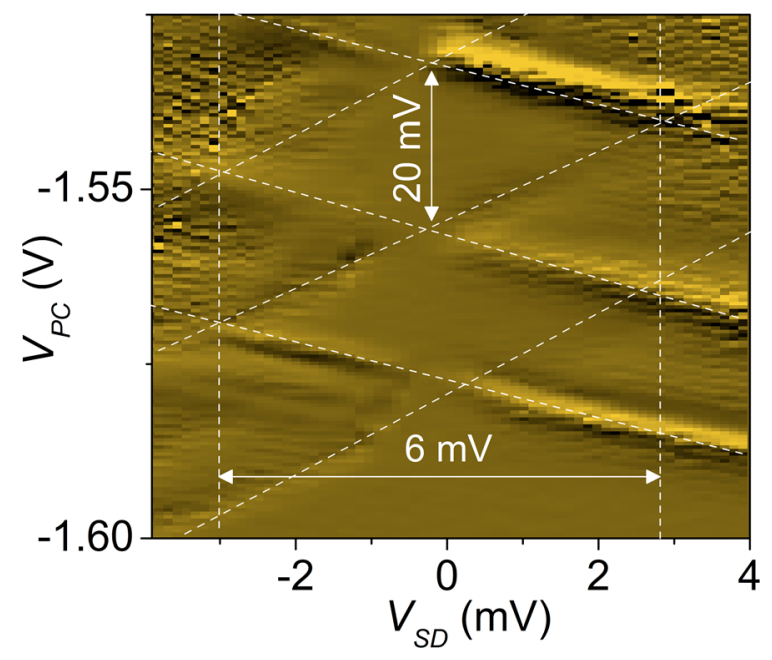

FIG. 3. Intensity plot of $-d^{2} I_{S D} / d V_{S D}^{2}$ close to pinch-off region. The white dashed lines are guide to the eye depicting the diamond-shape of stability diagram. the QD gate and the $\operatorname{dot} C_{Q D G}^{S D} \sim 1.1 \times 10^{-17} \mathrm{~F}$. If we solve the Laplace equation for a system QD gate-SD with the intention to get this capacitance-then the small dot should have the diameter of only $\sim 35 \mathrm{~nm} .{ }^{17}$ The estimations imply that such a dot would contain from 3 to 30 electrons. We modelled the potential profile created by the QD and PC gates. It has a saddle shape, without any local minima. In Ref. 15, phosphorus impurity atoms were intentionally embedded in the quasi-1D channel. A small dots consisting of a few electrons were formed around impurity atoms, which resulted in $\mathrm{CB}$ peaks in the conductance. The period, however, was irregular compared to our experiments. Usually aperiodic oscillations are observed when two or more coupled dots are formed in the conductance channel. ${ }^{18}$ In all of our samples, the oscillations were nearly periodic; moreover, we do not expect impurities in the channel because of modulation doping of our hetero-structure. We suggest a following explanation of the SD origin. It is known that monolayer roughness of quantum well interfaces gives rise to modulation of the bound state energies. ${ }^{19}$ The latter can be as large as $\sim 0.5 \mathrm{meV}$ in our hetero-structure. A lateral scale of this roughness can be as large as $10 \mathrm{~nm}$, depending on parameters of growth and substrate misorientation. The SD in the PC channel can be randomly formed because of this roughness, which would give rise to periodic oscillations of conductance.

\section{OPERATION OF QUANTUM DOT-SINGLE ELECTRON TRANSISTOR DEVICE}

Additional information about QD-SET and QD-PC devices is acquired from experiments with the SET. Particularly, the SET enables to identify region where the QD is formed. In experiment, we have applied constant voltage of $1 \mathrm{mV}$ between source and drain of SET, and probed the current. When $V_{P C}$ is fixed the SET current oscillates with $V_{Q D}$, see Fig. 4(a). The period of oscillations has a sharp transition from 81 to $5.5 \mathrm{mV}$ at $V_{Q D} \sim-1.2 \mathrm{~V}$ due to building up an extra capacitance between the QD gate and the SET through the newly formed QD, see Eq. (1) below. We measure the map of $\mathrm{CB}$ oscillations by sweeping $V_{Q D}$ from -1.0 to $-1.4 \mathrm{~V}$ at fixed $V_{P C}$, see Fig. 4(b). There are two regions with different oscillation periods of SET at the map. The boundary marks formation of the QD. The QD-SET device can be used as terahertz detector in the vicinity of the boundary. ${ }^{8}$ We translate this boundary to Fig. 2(a) as a white dashed line in order to show the region where the QD is formed. The white dashed line in Fig. 4(b) is a pinch-off boundary of the PC, taken from Fig. 2(a). The PC channel is pinched-off below the line in Fig. 4(b). One can expect effect of charging of the SD in the vicinity of the dashed line of Fig. 4(b). The effect is determined by the capacitance $C_{S D}^{S E T}$ between the SET and the SD. We estimate $C_{S D}^{S E T} \sim 1 \times 10^{-17}$ $\mathrm{F}$ by solving the Laplace equation. The effect of charging of the small dot would be seen as a shift of the SET's CB peak position by $\sim 0.7 \mathrm{mV}$. Observation of this shift is unfortunately beyond the experimental accuracy, because the period of charging of the $\mathrm{SD}$ is large compared to the period of $\mathrm{CB}$ of the SET, $14 \mathrm{mV}$ vs $5.5 \mathrm{mV}$. A small shift of the CB peaks 

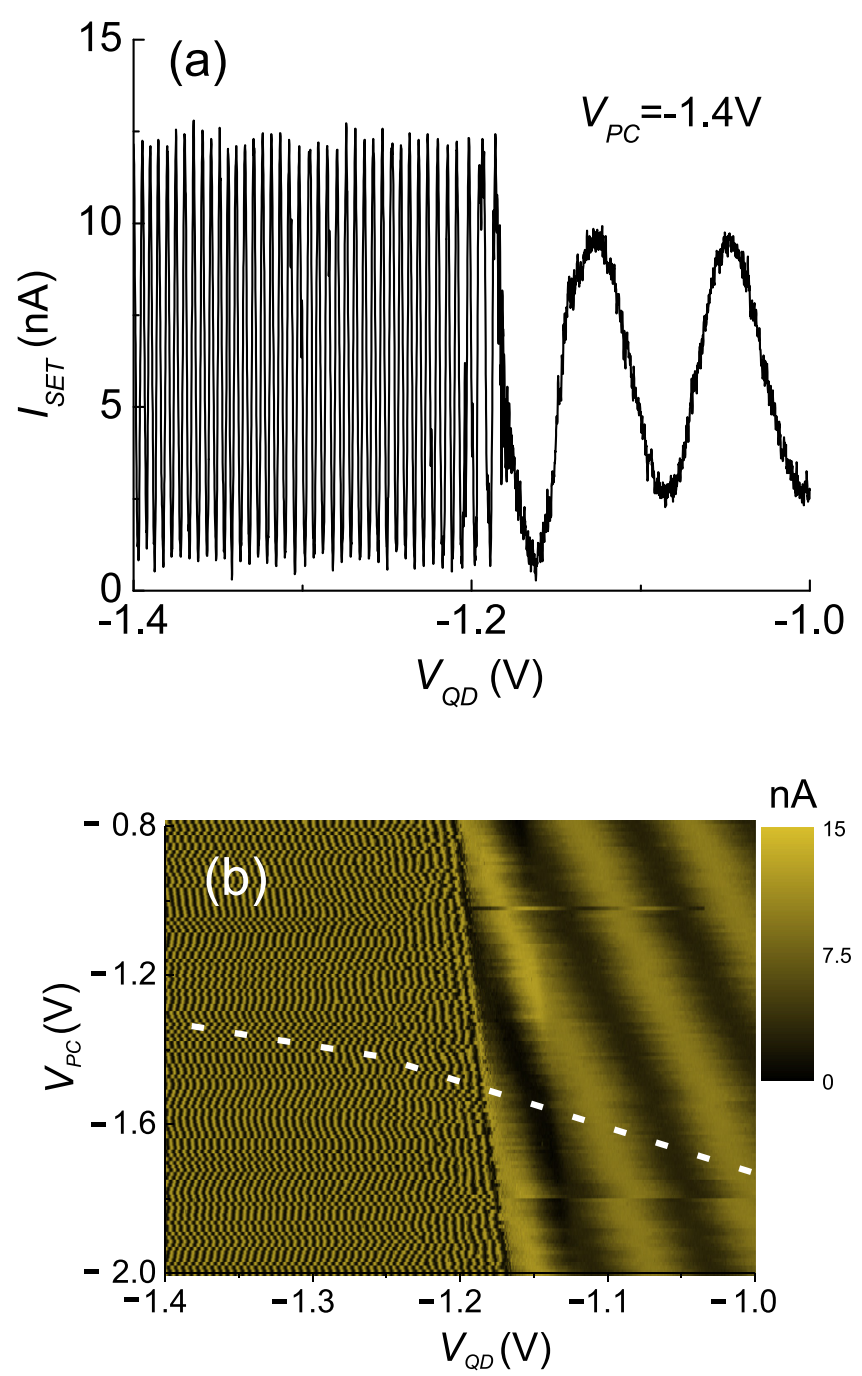

FIG. 4. (a) CB oscillations of the SET current. Period of CB oscillations changes from 81 to $5.5 \mathrm{mV}$ when QD is decoupled from the reservoirs. (b) Intensity plot of the $\mathrm{CB}$ oscillations in coordinates of $V_{Q D}$ and $V_{P C}$. The dashed white line indicates the pinch-off boundary of the PC.

of the SET over a few periods is hampered by the arbitrary fluctuation of the $\mathrm{CB}$ position.

In order to complete analysis, we present capacitances of the electrostatic model, see Table I. In the table, symbol $\Sigma$ denotes the total capacitance of a corresponding element to the environment. When populating the table we use period of $\mathrm{CB}$ oscillations of $\mathrm{SET}$ in order to find $C_{\mathrm{QDG}}^{S E T}, C_{\mathrm{PCG}}^{S E T}$, and $C_{\mathrm{SET}}^{Q D}$. In order to calculate $C_{\mathrm{SET}}^{Q D}$, we apply potential to $2 \mathrm{DEG}$ with zero potential at the QD gate. $C_{\Sigma}^{S E T}$ was calculated from the charging energy of SET. Capacitances of the point contact to the QD and SET are somewhat artificial, because the

TABLE I. Capacitances of the electrostatic circuit model of the device. Symbol $\Sigma$ denotes the total capacitance of a corresponding element to the environment.

\begin{tabular}{lcccccc}
\hline \hline & SET & QD & PC & $\Sigma$ & QDG & PCG \\
\hline SET & - & $75 \mathrm{aF}$ & $21 \mathrm{aF}$ & $0.4 \mathrm{fF}$ & $2 \mathrm{aF}$ & $0.24 \mathrm{aF}$ \\
QD & $75 \mathrm{aF}$ & - & $56 \mathrm{aF}$ & $0.25 \mathrm{fF}$ & $0.27 \mathrm{fF}$ & $24 \mathrm{aF}$ \\
PC & $21 \mathrm{aF}$ & $56 \mathrm{aF}$ & - & $50 \mathrm{aF}$ & $50 \mathrm{aF}$ & $50 \mathrm{aF}$ \\
\hline \hline
\end{tabular}

point contact always has a good coupling to the 2DEG. We found $C_{\mathrm{PC}}^{S E T} \sim 21 \mathrm{aF}$ using $\mathrm{CB}$ oscillations of the SET when potential is applied to the $2 \mathrm{DEG}$ with the $\mathrm{PC}$ almost pinched off. By solving the Laplace equation for the $\mathrm{PC}$ of $0.1 \times 0.1 \mu \mathrm{m}^{2}$ size, we have got $C_{\mathrm{PC}}^{S E T} \sim 30 \mathrm{aF}$, which is close to the experimental value. The remaining capacitances have been found from numerical solution of Laplace equation. One can do a consistency check of the capacitances by calculating the effective capacitance between the SET and the QD gate, when the QD is strongly coupled and isolated from the reservoirs. The difference in effective capacitance is seen as a change of period of the SET CB oscillations, Fig. 4(a). In the case of strong coupling, when $C_{\Sigma}^{Q D} \rightarrow \infty$, the effective capacitance is equal to $C_{Q D G}^{S E T}$. Once the QD becomes isolated from the reservoirs, the capacitance becomes

$$
C_{e f f} \approx C_{Q D G}^{S E T}+\frac{C_{Q D G}^{Q D} C_{\Sigma}^{S E T} C_{S E T}^{Q D}}{C_{\Sigma}^{Q D}+C_{Q D G}^{Q D} C_{\Sigma}^{S E T}} .
$$

From the experiment, we found $C_{\text {eff }}=2.9 \times 10^{-17} \mathrm{~F}$. The estimated value, $\sim 3.1 \times 10^{-17} \mathrm{~F}$, is very close to the experimental one.

\section{CHARGE SENSITIVITY OF QD-SET AND QD-PC DEVICES}

The sensitivities of the SET and PC to charge fluctuation at the QD are determined by the trans-conductance, $d G / d V_{g}$, and the capacitive coupling between the PC and the SET to the QD, $V_{g}$ being the voltage applied to the gate forming the $\mathrm{PC}$ or the SET gate. One can combine two factors by introducing the sensitivity of the source-drain current to charge variation at the $\mathrm{QD}, d I / d Q$. Once the current noise in the system, $\delta I$, is known, the detectable level of QD charge excitation would be $\delta Q=\delta I /\left(\frac{d I}{d Q}\right)$. We found a moderate sensitivity of the PC to charge excitation in region $b$ of Fig. 2, $d I / d Q \sim 4 \times 10^{6} \mathrm{~A} / \mathrm{C}$. Our set up has $\delta I \sim 3 \mathrm{pA}$ in a bandwidth $\sim 1 \mathrm{kHz}$. The PC is then able to detect excitation of few electrons, $\delta Q \sim 4 e$, in/out of the QD. The sensitivity is enhanced in a region where the $\mathrm{CB}$ oscillations are present. Typically, the maxima of $d I / d Q$ are higher by $\sim 15 \%$ in these regions. The drawback is that the sensitivity becomes dependent on the operation points: it is reduced at the extremes of the $\mathrm{CB}$ oscillations and it is enhanced at the slopes. This makes the photo-detector to be less stable in operation. The sensitivity of the SET readout is only slightly higher, $d I / d Q \sim 10^{7} \mathrm{~A} / \mathrm{C}$. It is constant along the boundary where the QD is formed. There is $25 \%$ variation of sensitivity depending on the operation point of SET itself. It follows from the analysis that the SET should be able to detect excitation of $\delta Q \sim e$ in a bandwidth of $1 \mathrm{kHz}$. Indeed, the excitations of individual electrons are clearly seen in our QD-SET device, they produce spikes close to pinch-off region in Fig. 4(a).

\section{CONCLUSIONS}

In summary, we study the operation of the QD-SET-PC device. We describe the system with the electrostatic model 
and determine a set of corresponding capacitances. The device is able to detect charge excitations of the QD with an accuracy of less than one electron for the SET-QD and a few electrons for PC-QD system. There are periodic oscillations of the PC conductance close to pinch-off. We believe that the oscillations are result of the Coulomb blockade of electron tunnelling in a quasi-1D channel, due to the formation of a small dot of electrons, 35-100 nm size. The small dot can be formed because of quantum well interface roughness.

\section{ACKNOWLEDGMENTS}

We acknowledge stimulating discussions with V. Gurtovoi. This work is supported by the Solution Oriented Research for Science and Technology (SORST) from the Japan Science and Technology (JST), EPSRC Grants EP/F005482/1, EP/G061432/1, and EU Integrated Project, TERAEYE.

${ }^{1}$ B. J. van Wees, H. van Houten, C. W. J. Beenakker, J. G. Williamson, L. P. Kouwenhoven, D. van der Marel, and C. T. Foxon, Phys. Rev. Lett. 60, 848 (1988)

${ }^{2}$ S. Foletti, H. Bluhm, D. Mahalu, V. Umansky, and A. Yacoby, Nat. Phys. 5, 903-908 (2009).

${ }^{3}$ J. C. Chen, Y. Lin, K. Ting Lin, T. Ueda, and S. Komiyama, Appl. Phys. Lett. 94, 012105 (2009).
${ }^{4}$ D. Taubert, M. Pioro-Ladriere, D. Schroer, D. Harbusch, A. S. Sachrajda, and S. Ludwig, Phys. Rev. Lett. 100, 176805 (2008).

${ }^{5}$ S. Nadj-Perge1, S. M. Frolov, E. P. A. M. Bakkers, and L. P. Kouwenhoven, Nature 468, 1084 (2010).

${ }^{6}$ U. Gasser, S. Gustavsson, B. Kung, K. Ensslin, T. Ihn, D. C. Driscoll, and A. C. Gossard, Phys. Rev. B 79, 035303 (2009).

${ }^{7}$ M. Field, C. G. Smith, M. Pepper, D. A. Ritchie, J. E. F. Frost, G. Jones, and D. G. Hasko, Phys. Rev. Lett. 70, 1311 (1993).

${ }^{8}$ H. Hashiba, V. Antonov, L. Kulik, S. Komiyama, and C. Stanley, Appl. Phys. Lett. 85, 6036 (2004).

${ }^{9}$ S. Pelling, R. Davis, L. Kulik, A. Tzalenchuk, S. Kubatkin, T. Ueda, S. Komiyama, and V. N. Antonov, Appl. Phys. Lett. 93, 073501 (2008).

${ }^{10}$ G. J. Dolan, Appl. Phys. Lett. 31, 337 (1977).

${ }^{11}$ T. Rejec and Y. Meir, Nature 442, 900 (2006).

${ }^{12}$ A. Kristensen, H. Bruus, A. E. Hansen, J. B. Jensen, P. E. Lindelof, C. J. Marckmann, J. Nygård, C. B. Sørensen, F. Beuscher, A. Forchel, and M. Michel Phys. Rev. B 62, 10950 (2000).

${ }^{13}$ A. C. G. T.-M. Chen, M. Pepper, I. Farrer, and D. A. Ritchie, Appl. Phys. Lett. 93, 032102 (2008).

${ }^{14}$ C. de Graaf, J. Caro, S. Radelaar, V. Lauer, and K. Heyers, Phys. Rev. B 44, 9072 (1991).

${ }^{15}$ M. Tabe, D. Moraru, M. Ligowski, M. Anwar, R. Jablonski, Y. Ono, and T. Mizuno, Phys. Rev. Lett. 105, 016803 (2010).

${ }^{16}$ L. P. Kouwenhoven, F. W. J. Hekking, B. J. van Wees, C. J. P. M. Harmans, C. E. Timmering, and C. T. Foxon, Phys. Rev. Lett. 65, 361-364 (1990).

${ }^{17}$ We use 3D FLEXPDE simulation package of PDE solutions Inc.

${ }^{18}$ A. V. Danilov, D. S. Golubev, and S. E. Kubatkin, Phys. Rev. B 65, 125312 (2002).

${ }^{19}$ R. Grousson, V. Voliotis, N. Grandjean, J. Massies, M. Leroux, and C. Deparis, Phys. Rev. B 55, 5253 (1997). 